\title{
Finding binomials in polynomial ideals
}

\author{
Anders Jensen ${ }^{1}$, Thomas Kahle ${ }^{2^{*}}$ and Lukas Katthän ${ }^{3}$
}

${ }^{*}$ Correspondence:

thomas.kahle@ovgu.de

2Otto-von-Guericke Universität

Magdeburg, Germany

Full list of author information is

available at the end of the article

\begin{abstract}
We describe an algorithm which finds binomials in a given ideal / $\subset \mathbb{Q}\left[x_{1}, \ldots, x_{n}\right]$ and in particular decides whether binomials exist in / at all. Binomials in polynomial ideals can be well hidden. For example, the lowest degree of a binomial cannot be bounded as a function of the number of indeterminates, the degree of the generators, or the Castelnuovo-Mumford regularity. We approach the detection problem by reduction to the Artinian case using tropical geometry. The Artinian case is solved with algorithms from computational number theory.
\end{abstract}

Keywords: Algorithm, Binomial detection, Binomial ideal, Tropical geometry

Mathematics Subject Classification: Primary: 68W99, Secondary: 11R04, 11Y16, 11Y40, 13P05, 13P99, 14T05, 68W30

\section{Background}

The goal of this paper is to prove the following result.

Theorem 1 There is a deterministic algorithm that, given generators, decides whether an ideal in the polynomial ring $\mathbb{Q}\left[x_{1}, \ldots, x_{n}\right]$ contains nonzero binomials.

Theorem 1 answers a fundamental question in computational algebra, but we envision that it will also be useful for applications. To name just a few, when implementing mesoprimary decomposition of binomial ideals [11], a test for binomials is necessary. In the theory of retractions of polytopal algebras, [3, Conjecture B] is connected to the existence of binomials and monomials in the kernels of certain maps (albeit after a graded automorphism of the ambient ring). In [20], Sontag argues that polynomials with few terms in an ideal yield the best restrictions on the possible sign patterns of changes that a steady state of a chemical reaction network can undergo under perturbation. Theorem 1 can also be seen as a first step to the broader problem of deciding whether an ideal contains a sparse polynomial, or finding the sparsest polynomial. For example, Jürgen Herzog suggested the problem of determining the length of the shortest polynomial in a standard determinantal ideal.

It does not seem possible to prove Theorem 1 by standard arguments using Gröbner bases. For example, the ideal $\left\langle x^{2}+x+1\right\rangle \subset \mathbb{Q}[x]$ contains $x^{3}-1$, but its generator, trivially, is a universal Gröbner basis. Moreover, the lowest degree binomials in an ideal need not satisfy a general upper degree bound in terms of common invariants such as Castelnuovo-Mumford regularity or primary decomposition.

(c) The Author(s) 2017. This article is distributed under the terms of the Creative Commons Attribution 4.0 International License (http://creativecommons.org/licenses/by/4.0/), which permits unrestricted use, distribution, and reproduction in any medium, provided you give appropriate credit to the original author(s) and the source, provide a link to the Creative Commons license, and indicate if changes were made. 
Example 2 For any $n \in \mathbb{N}$, let $\left.I=\left\langle(x-z)^{2}, n x-y-(n-1) z\right)\right\rangle \subset \mathbb{Q}[x, y, z]$. The Castelnuovo-Mumford regularity of $I$ is 2 and it is primary over $\langle x-z, y-z\rangle$. The binomial $x^{n}-y z^{n-1}$ is contained in $I$ because an elementary computation shows that

$$
x^{n}-y z^{n-1}=\sum_{k=0}^{n-2}(n-k-1) x^{k} z^{n-k-2}(x-z)^{2}+z^{n-1}(n x-y-(n-1) z) \in I .
$$

There is no binomial of degree less than $n$ in $I$. To see this, consider the differential operators $D_{1}=\partial_{x}+n \partial_{y}$ and $D_{2}=(1-n) \partial_{y}+\partial_{z}$. Any element $f \in I$ satisfies $f(1,1,1)=$ $0,\left(D_{1} f\right)(1,1,1)=0$ and $\left(D_{2} f\right)(1,1,1)=0$ as both generators have this property. Assume that $I$ contains the binomial $f=x^{u}-\lambda y^{v}$. First, note that $f(1,1,1)=0$ implies that $\lambda=1$. Further, $\left(D_{1} f\right)(1,1,1)=0$ and $\left(D_{2} f\right)(1,1,1)=0$ give two linear conditions on the vector $u-v$, which imply that $u-v=m(n,-1,1-n)$ for some $m \in \mathbb{Z}$. By exchanging $u$ and $v$ we may assume that $m>0$, so it follows that $f=x^{m n}-y^{m} z^{m(n-1)}$. In particular, there is no binomial of degree less than $n$ in $I$.

Our approach to Theorem 1 can be summarized as follows. Given an ideal $I \subset$ $\mathbb{Q}\left[x_{1}, \ldots, x_{n}\right]$, we pass to its Laurent extension $J=I \mathbb{Q}\left[x_{1}^{ \pm}, \ldots, x_{n}^{ \pm}\right]$, which contains binomials if and only if $I$ contains binomials (Lemma 5). We then show in Section 3 that there exists an ideal $J^{\prime} \subseteq \mathbb{Q}\left[y_{1}^{ \pm}, \ldots, y_{m}^{ \pm}\right]$such that $\mathbb{Q}\left[y_{1}^{ \pm}, \ldots, y_{m}^{ \pm}\right] / J^{\prime}$ is Artinian, and the sets of binomials in $J$ and $J^{\prime}$ can easily be computed from each other (Proposition 12 and Theorem 13). This reduction is achieved by means of tropical geometry. The Artinian case is easier since the (images of the) indeterminates $y_{1}, \ldots, y_{m}$ in $\mathbb{Q}\left[y_{1}^{ \pm}, \ldots, y_{m}^{ \pm}\right] / J^{\prime}$ have matrix representations that commute. This leads to the constructive membership problem for commutative matrix (semi)groups [1], which is already solved (see [9] for a survey). The completed algorithm appears as Algorithm 22 in Section 5.

\section{Related work and variations of the problem}

The question whether an ideal is a binomial ideal, that is, whether it can be generated by binomials alone, can be decided by computing a reduced Gröbner basis [6, Corollary 1.2]. In the case of a homogeneous ideal one can even do it with linear algebra only [5, Proposition 3.7].

Moreover, deciding for given monomials $\mathbf{x}^{u}$ and $\mathbf{x}^{v}$ whether there exists some scalar $\lambda$ such that $\mathbf{x}^{u}-\lambda \mathbf{x}^{v}$ is contained in a given ideal $I$ is also not too difficult. For this problem, it suffices to compute the unique normal forms of $\mathbf{x}^{u}$ and $\mathbf{x}^{v}$ modulo a Gröbner basis of $I$ and check whether they are scalar multiples of each other. Using this observation, one can decide whether $I$ contains a binomial of a given degree by brute force. However, this approach cannot be used to prove Theorem 1 , because there is no a priori degree bound on a binomial in $I$ (cf. Example 2).

It appears that primary decomposition is not helpful for the problem at hand. For example, the ideal $\langle(x-y)(z-w)\rangle=\langle x-y\rangle \cap\langle z-w\rangle$ does not contain a binomial, even though its minimal primes are generated by binomials.

Finally, the detection of monomials in a polynomial ideal is quite simple using ideal quotients: an ideal $I \subset \mathbb{Q}\left[x_{1}, \ldots, x_{n}\right]$ contains a monomial if and only if $\left(\left(\cdots\left(I: x_{1}^{\infty}\right) \cdots\right)\right.$ : $\left.x_{n}^{\infty}\right)=\left(I:\left(x_{1} \cdots x_{n}\right)^{\infty}\right)=\mathbb{Q}\left[x_{1}, \ldots, x_{n}\right]$. The colon ideals $\left(I: x_{i}^{\infty}\right)$ can readily be computed with Gröbner bases [8, Section 1.8.9]. It was discovered several times that extensions of this yield all monomials (see [17], [19, Algorithm 4.4.2], or [13, Tutorial 50]). 


\section{Binomials in ideals}

As in the case of binomial ideals, it is more convenient to work not only with the binomials in an ideal, but with the entire subspace they generate. Throughout this section, let $\mathbb{K}$ be any fixed field and denote by $S=\mathbb{K}\left[x_{1}, \ldots, x_{n}\right]$ the polynomial ring in $n$ indeterminates with coefficients in $\mathbb{K}$. We occasionally use the notation $\mathbf{x}^{a}:=\prod_{i} x_{i}^{a_{i}}$ for $a=\left(a_{1}, \ldots, a_{n}\right) \in$ $\mathbb{N}^{n}$.

Definition 3 Let $I \subset S$ be an ideal. The binomial part $\operatorname{Bin}(I)$ of $I$ is the $\mathbb{K}$-subspace of $I$ spanned by all binomials in $I$.

Proposition 4 The binomial part of any ideal is a binomial ideal.

Proof Let $I \subset S$ be an ideal and $B \subset I$ its binomial part. Then every element $b \in B$ is a linear combination of binomials. Multiplying it with an arbitrary $f \in S$ yields some linear combination of monomial multiples of the binomials in $b$. Since $I$ is an ideal, those monomial multiples are contained in $B$ too and so is $f b$. Thus, $B$ is an ideal. Moreover, the ideal $B$ is binomial, since an ideal is in particular generated by any set that generates it as a vector space.

By the same argument, a binomial ideal is as a vector space spanned by the binomials it contains. In particular, a binomial ideal equals its binomial part.

We now discuss ring extensions in this context. Denote by $T=\mathbb{K}\left[x_{1}^{ \pm}, \ldots, x_{n}^{ \pm}\right]$the Laurent polynomial ring corresponding to $S$. We extend the notion of $\operatorname{Bin}(I)$ to this ring in the natural way.

Lemma 5 For any ideal $I \subset S$, it holds that $\operatorname{Bin}(I T)=\operatorname{Bin}(I) T$. In particular, I contains a binomial if and only if the extension of I to $T$ contains a binomial. Moreover, if ( $I$ : $\left.x_{1} \cdots x_{n}\right)=I$, then $\operatorname{Bin}(I)=\operatorname{Bin}(I T) \cap S$.

Proof The inclusion " $\supseteq$ " is clear because $I \subset I T$. For the other inclusion, note that any binomial in $I T$ can be multiplied with a monomial to obtain a binomial in $I$. The last statement follows from the fact the hypothesis implies that $I=I T \cap S$.

The binomial part of a proper ideal $I \subset T$ is determined by a lattice $L \subset \mathbb{Z}^{n}$ and a homomorphism $\phi: L \rightarrow \mathbb{K}^{\times}$(called a partial character in [6]). According to [6, Theorem 2.1], the binomial part of $I$ is the binomial ideal $\left\langle\mathbf{x}^{m}-\phi(m): m \in L\right\rangle$.

Remark 6 For ideals in polynomial rings, a partial character is not a sufficient data structure to store all binomials, for there typically exist associated primes containing indeterminates. When passing to the Laurent ring, these associated primes are annihilated and many new binomials may be created. For example, $\left\langle x-y, x^{2}, x y, y^{2}\right\rangle \subset \mathbb{K}[x, y]$ extends to the entire Laurent ring $\mathbb{K}\left[x^{ \pm}, y^{ \pm}\right]$, while for

$$
\left\langle x^{2}-y^{2}, x^{3}-x^{2} y\right\rangle
$$

the information about the index two lattice of binomials of degree two is lost by the appearance of $x-y$, which generates the extension to the Laurent ring. However, Lemma 5 guarantees that the extension to the Laurent ring only yields new binomials if binomials are present in the original ideal. 
Lemma 7 Let $\mathbb{K}^{\prime} / \mathbb{K}$ be any field extension and $T^{\prime}:=T \otimes_{\mathbb{K}} \mathbb{K}^{\prime}$ be the Laurent polynomial ring over $\mathbb{K}^{\prime}$. Then, for any ideal $I \subset T$, it holds that

$$
\operatorname{Bin}\left(I T^{\prime}\right)=\operatorname{Bin}(I) T^{\prime} .
$$

In particular,

- I contains a binomial if and only if IT' contains a binomial, and

- $\operatorname{Bin}(I)=\operatorname{Bin}\left(I T^{\prime}\right) \cap T$.

Proof As above, the inclusion " $\supseteq$ " is clear because $I \subset I T^{\prime}$. Moreover, the claim is clear if $I T^{\prime}=T^{\prime}$, so we may assume that $I$ is a proper ideal. Suppose now that $I T^{\prime}$ contains the binomial $\mathbf{x}^{u}-\lambda \mathbf{x}^{v}$ with $u, v \in \mathbb{Z}^{n} \backslash\{0\}, \lambda \in \mathbb{K}^{\prime}$. Then there exists an expression

$$
\mathbf{x}^{u}-\lambda \mathbf{x}^{v}=\sum_{i} \lambda_{i} \mathbf{x}^{v_{i}} f_{i}
$$

with $v_{i} \in \mathbb{Z}^{n}, \lambda_{i} \in \mathbb{K}^{\prime}$ and $f_{i} \in I$. When $u, v, v_{i}$ and $f_{i}$ are fixed, (2.1) can be interpreted as a system of linear equations in the unknowns $\lambda$ and $\lambda_{i}$. This system has a solution over $\mathbb{K}^{\prime}$, and because its coefficients are in $\mathbb{K}$, it also has a solution over $\mathbb{K}$. Moreover, there is only one value possible for $\lambda$, because otherwise $\mathbf{x}^{\nu} \in I T^{\prime}$ and thus $I T^{\prime}=T^{\prime}$. Hence, $\lambda \in \mathbb{K}$ and $\mathbf{x}^{u}-\lambda \mathbf{x}^{v} \in I$. Every element of $\operatorname{Bin}(I)$ can be written as a linear combination of binomials of this form, and the claim follows.

For the last claim, we only need to show the inclusion $\operatorname{Bin}(I) \supseteq \operatorname{Bin}\left(I T^{\prime}\right) \cap T$. Choose a $\mathbb{K}$-basis $\mathcal{B}$ of $\operatorname{Bin}(I)$. By the argument above, it is also a $\mathbb{K}^{\prime}$-basis of $\operatorname{Bin}\left(I T^{\prime}\right)$, and thus, every binomial $b \in \operatorname{Bin}\left(I T^{\prime}\right)$ has a unique expansion in this basis. Hence, $b$ lies in $T$ if and only if its coefficients in this expansion lie in $\mathbb{K}$. But the latter implies that $b \in \operatorname{Bin}(I)$.

Remark 8 Let $I \subset \mathbb{Q}\left[x_{1}^{ \pm}, \ldots, x_{n}^{ \pm}\right]$be an ideal. Our algorithms usually construct the binomial part of the extension $I \mathbb{K}\left[x_{1}^{ \pm}, \ldots, x_{n}^{ \pm}\right]$to the Laurent ring with coefficients in a finite extension $\mathbb{K}$ of $\mathbb{Q}$. Lemma 7 guarantees that this yields a determination of the binomial part of an ideal $I \subset \mathbb{Q}\left[x_{1}^{ \pm}, \ldots, x_{n}^{ \pm}\right]$, because

$$
\operatorname{Bin}(I)=\operatorname{Bin}\left(I \mathbb{K}\left[x_{1}^{ \pm}, \ldots, x_{n}^{ \pm}\right]\right) \cap \mathbb{Q}\left[x_{1}^{ \pm}, \ldots, x_{n}^{ \pm}\right] .
$$

Example 9 Remark 8 shows that the binomial part is preserved when extending the coefficient field and then contracting back. It is not generally true that binomial parts survive contraction followed by extension. For example, $\langle x-\sqrt{2}\rangle \subset \mathbb{Q}(\sqrt{2})[x]$ contracts to $\left\langle x^{2}-2\right\rangle \subset \mathbb{Q}[x]$ which in turn extends to $\left\langle x^{2}-2\right\rangle \subset \mathbb{Q}(\sqrt{2})[x]$ by Lemma 7 .

\section{Reducing to the Artinian case via tropical geometry}

Our eventual goal it to compute $\operatorname{Bin}(I)$ for arbitrary ideals $I \subset \mathbb{Q}\left[x_{1}, \ldots, x_{n}\right]$. In this section, we use tropical geometry which means that we have to work with the extension of $I$ to the Laurent polynomial ring. By Lemma 5 , this is sufficient to determine whether $\operatorname{Bin}(I)$ is empty or not. Moreover, if $\left(I: x_{1} \cdots x_{n}\right)=I$ then our methods determine all of $\operatorname{Bin}(I)$.

If $I$ is a prime ideal over an algebraically closed field, then tropical geometry yields a complete answer: the ideal contains binomials if and only if the tropical variety is contained in a tropical hypersurface of a binomial, i.e., in an ordinary hyperplane (Corollary 14). In fact, one implication is immediate from the following definitions. The algebraically 
closedness assumption is easy to relax, but if the ideal is not prime the tropical variety alone does not reveal binomial containment as the following example demonstrates.

Example 10 The principal ideal $\langle(x-1)(x-2)\rangle \subset \mathbb{C}[x]$ has tropical variety $\{0\}$. It cannot contain a binomial, since such binomial would have roots with different moduli, which binomials cannot have.

However, expanding on the idea from the prime case we can use tropical geometry to reduce binomial detection to the case of ideals with Artinian quotients, which we call Artinian ideals for short. The results in this section hold for more general coefficient fields than $\mathbb{Q}$. To this end, let $\mathbb{K}$ be a field and $\overline{\mathbb{K}}$ its algebraic closure. The reader interested only in Theorem 1 can mentally replace $\mathbb{K}$ by $\mathbb{Q}$. It is notationally convenient to understand the Laurent ring $\mathbb{K}\left[x_{1}^{ \pm}, \ldots, x_{n}^{ \pm}\right]$as the group ring $\mathbb{K}\left[\mathbb{Z}^{n}\right]$. This is the ambient ring for this section.

Definition 11 Let $L$ be an integer lattice, $M$ the dual lattice and $I \subset \mathbb{K}[L]$ an ideal. For $\omega \in \mathbb{Q} \otimes M$ the initial form $\operatorname{in}_{\omega}(f)$ of a polynomial $f=\sum_{\nu} c_{\nu} \mathbf{x}^{v}$ is the sum of terms $c_{\nu} \mathbf{x}^{\nu}$ for which $\langle\omega, v\rangle$ is maximal. For an ideal $I \subset \mathbb{K}[L]$, the initial ideal of $I$ with respect to $\omega$ $\operatorname{is~}_{\omega}(I)=\left\langle\operatorname{in}_{\omega}(f): f \in I\right\rangle$. The tropical variety of $I$ is

$$
T(I)=\left\{\omega \in \mathbb{Q} \otimes M: \operatorname{in}_{\omega}(I) \neq \mathbb{K}[L]\right\} .
$$

If $L=\mathbb{Z}^{n}$ and $I$ is homogeneous, the definition can be stated in terms of initial ideals of homogeneous ideals in a polynomial ring. Then $T(I)$ is the support of a subfan of the Gröbner fan of $I \cap \mathbb{K}\left[\mathbb{N}^{n}\right]$, a fan in $\mathbb{Q}^{n}$ that has one cone for each initial ideal of $I \cap \mathbb{K}\left[\mathbb{N}^{n}\right]$. Since $\operatorname{in}_{\omega}(I) \neq \mathbb{K}[L]$ can be decided by Gröbner bases, the definition can be turned into an algorithm computing tropical varieties [2].

If a polynomial $f$ is a binomial, then $T(\langle f\rangle)$ is a hyperplane (or empty) and the Newton polytope of $f$ is a line segment orthogonal to $T(\langle f\rangle)$. The inclusion $T(\langle f\rangle) \supset T(I)$ for $f \in I$ implies that if $I$ contains a binomial $f$, then the Newton polytope of $f$ must be perpendicular to $T(I)$. Thus, if $I$ contains a binomial then also $I \cap \mathbb{K}\left[T(I)^{\perp} \cap L\right]$ contains a binomial. The following proposition extends this to all of $\operatorname{Bin}(I)$.

Proposition 12 Let $L$ be a lattice and $I \subset \mathbb{K}[L]$ an ideal. Then

$$
\operatorname{Bin}(I)=\operatorname{Bin}\left(I \cap \mathbb{K}\left[T(I)^{\perp} \cap L\right]\right) \mathbb{K}[L] .
$$

Proof Let $f \in I$ be a binomial generator of the left-hand side. Then the Newton polytope of $f$ is perpendicular to $T(I)$, meaning that $\mathbf{x}^{u} f \in I \cap \mathbb{K}\left[T(I)^{\perp} \cap L\right]$ for some $u \in L$. Hence, $f=\mathbf{x}^{u} f \mathbf{x}^{-u} \in \operatorname{Bin}\left(I \cap \mathbb{K}\left[T(I)^{\perp} \cap L\right]\right) \mathbb{K}[L]$. The other containment is clear since $I \cap \mathbb{K}\left[T(I)^{\perp} \cap L\right] \subset I$.

The lattice $T(I)^{\perp} \cap L$ is a saturated lattice in $L$, and therefore, after a multiplicative change of coordinates, we may assume that $\left(T(I)^{\perp} \cap L\right) \times\{0\}^{n-m}=\mathbb{Z}^{m} \times\{0\}^{n-m} \subset \mathbb{Z}^{n}=L$ with $m=\operatorname{dim}\left(T(I)^{\perp}\right)$. Generators for $I \cap \mathbb{K}\left[T(I)^{\perp} \cap L\right]$ can then be computed by the elimination $I \cap \mathbb{K}\left[x_{1}^{ \pm}, \ldots, x_{m}^{ \pm}\right]$. This can be reduced to a Gröbner basis computation in the polynomial ring by first passing to the saturation $\left(I:\left(x_{m+1} \cdots x_{n}\right)^{\infty}\right)$. The following theorem reduces the problem of deciding whether an ideal contains a binomial to the case of Artinian ideals. 
Theorem 13 Let $\mathbb{K}$ be any field, $L$ an integer lattice and $I \subset \mathbb{K}[L]$ an ideal. Then $I \cap$ $\mathbb{K}\left[T(I)^{\perp} \cap L\right]$ is an Artinian ideal in $\mathbb{K}\left[T(I)^{\perp} \cap L\right]$.

Proof Let $L^{\prime} \subseteq L$ be a saturated sublattice of $L$, and let $\iota: L^{\prime} \hookrightarrow L$ denote the inclusion map. It gives rise to a ring homomorphism $\mathbb{K}[\iota]: \mathbb{K}\left[L^{\prime}\right] \rightarrow \mathbb{K}[L]$, as well as to a dual map $\iota_{\mathbb{Q}}^{*}: L^{*} \otimes \mathbb{Q} \rightarrow\left(L^{\prime}\right)^{*} \otimes \mathbb{Q}$. We claim that

$$
\iota_{\mathbb{Q}}^{*}(T(I))=T\left(\mathbb{K}[\iota]^{-1}(I)\right) .
$$

To see this, note that $\mathbb{K}[l]$ induces a map $\phi:\left(\mathbb{K}^{\times}\right)^{n} \rightarrow\left(\mathbb{K}^{\times}\right)^{d}$ of tori, where $n=\operatorname{rank}(L)$ and $d=\operatorname{rank}\left(L^{\prime}\right)$. This map is monomial, because $\mathbb{K}[\iota]$ came from a map of lattices. Hence, [15, Corollary 3.2.13] implies that $\iota_{\mathbb{Q}}^{*}(T(I))=T(\overline{\phi(V(I))})$. On the other hand, by classical elimination theory it holds that $\overline{\phi(V(I))}=V\left(\mathbb{K}[\iota]^{-1}(I)\right)$, so taking the tropical variety yields the claim.

Now we turn to the proof of the theorem. For this, choose $L^{\prime}:=T(I)^{\perp} \cap L$. Then $\iota_{\mathbb{Q}}^{*}(T(I))=\{0\}$, because restricting a linear map to its kernel yields zero. On the other hand, it clearly holds that $\mathbb{K}[\iota]^{-1}(I)=I \cap \mathbb{K}\left[L^{\prime}\right]$, because $\mathbb{K}[\iota]$ is an inclusion. It follows that $\operatorname{dim} T\left(I \cap \mathbb{K}\left[L^{\prime}\right]\right)=0$. Finally, by the Bieri-Groves theorem [15, Theorem 3.3.5], this is also the dimension of the variety of $I \cap \mathbb{K}\left[L^{\prime}\right]$, and hence, this ideal is Artinian.

That our definition of tropical varieties is compatible with that in [15] follows from the Fundamental Theorem of Tropical Geometry [15, Theorem 3.2.3]. We employ [15, Corollary 3.2.13] when $\mathbb{K}$ is not algebraically closed, which is possible since extending the field does not affect the tropical varieties as they are defined via initial ideals that are computable via Gröbner bases. Similarly, if the field does not come with a non-trivial valuation (which is the case here), one may extend it to the field of generalized Puiseux series which has a non-trivial valuation. See also [15, Theorem 3.1.3].

The preceding theorem allows us to determine when a prime ideal contains a binomial.

Corollary 14 Let $L$ be an integer lattice and $I \subset \mathbb{K}[L]$ an ideal. If the extension $I \overline{\mathbb{K}}[L] \subset$ $\overline{\mathbb{K}}[L]$ of I to the algebraic closure is prime, then I contains a binomial if and only if $T(I)$ is contained in a hyperplane, i.e., $T(I)^{\perp} \neq\{0\}$.

Proof By Lemma 7 we may assume that $\mathbb{K}=\overline{\mathbb{K}}$. Moreover, by Proposition 12 we can consider $I^{\prime}:=I \cap \mathbb{K}\left[T(I)^{\perp} \cap L\right]$ instead of $I$. Now, if $T(I)^{\perp}=\{0\}$, then $I^{\prime}=\langle 0\rangle$ does not contain a binomial. On the other hand, if $T(I)^{\perp} \neq\{0\}$, then $I^{\prime}$ is a proper Artinian ideal. Hence, after choosing an identification $\mathbb{K}\left[T(I)^{\perp} \cap L\right]=\mathbb{K}\left[y_{1}^{ \pm}, \ldots, y_{m}^{ \pm}\right], I^{\prime}$ contains non-constant univariate Laurent polynomials in each of the $y_{i}$ and in particular a Laurent polynomial $f \in \mathbb{K}\left[y_{1}^{ \pm}\right]$. Because $\mathbb{K}$ is algebraically closed, we can factor $f$ as $f=c y_{1}^{a} \prod_{j}\left(y_{1}-\lambda_{j}\right)$ with $c, \lambda_{j} \in \mathbb{K}$ and $a \in \mathbb{Z}$. One factor is contained both in $\mathbb{K}\left[T(I)^{\perp} \cap L\right]$ and in $I$ (because $I$ is prime) and hence in $I^{\prime}$. Thus, $\operatorname{Bin}\left(I^{\prime}\right) \neq\{0\}$.

The intention is to apply Theorem 13 and Proposition 12 to reduce the computation of $\operatorname{Bin}(I)$ to the Artinian case for arbitrary $I \subset \mathbb{K}[L]$. To proceed, we must be able to compute the lattice $T(I)^{\perp} \cap L$. We formulate the following algorithms in the Laurent ring, but using saturations the necessary computations can be carried out in a polynomial ring.

It is possible to either compute the entire Gröbner fan or to apply the traversal strategy of [2] even if $I$ is not homogeneous to find $T(I)$, but both strategies have several drawbacks; 
a problematic one being that $T(I)$ can easily consist of millions of polyhedral cones. For this reason we offer an approach to directly compute $\operatorname{span}(T(I)) \subseteq \mathbb{Q}^{n}$. We will make the assumption that we have an algorithm with the following specification.

Algorithm 15 (Tropical Curve)

Input: Generators for an ideal $I \subset \mathbb{K}[L]$ defining $T(I)$ of dimension 1 .

Output: The rays of $T(I)$.

One such algorithm relying on tropical bases is presented in [2]. Another one relying on projections and elimination can be found in Andrew Chan's thesis [4]. We use Algorithm 15 to find a non-trivial vector in $T(I)$ as follows:

\section{Algorithm 16}

Input: Generators for an ideal $I \subset \mathbb{K}[L]$ with $d=\operatorname{dim}(I)>0$.

Output: A primitive vector in $T(I) \backslash\{0\}$.

1. Choosed -1 polynomials $u_{1}, \ldots, u_{d-1} \in \operatorname{span}_{\mathbb{K}}\left\{1, x_{1}, \ldots, x_{n}\right\}$ so that $\operatorname{dim}\left(I+\left\langle u_{1}, \ldots, u_{d-1}\right\rangle\right)=1$.

2. Compute $T\left(I+\left\langle u_{1}, \ldots, u_{d-1}\right\rangle\right)$ using Algorithm 15.

3. Return a primitive generator for one of the rays of $T\left(I+\left\langle u_{1}, \ldots, u_{d-1}\right\rangle\right)$.

The returned vector is indeed contained in $T(I)$, because $T\left(I+\left\langle u_{1}, \ldots, u_{d-1}\right\rangle\right) \subseteq T(I)$.

Remark 17 The dimension condition in the first step holds for a Zariski open subset of $\operatorname{span}_{\mathbb{K}}\left\{1, x_{1}, \ldots, x_{n}\right\}$. Therefore, these polynomials could be picked at random and checked to satisfy the dimension condition. There is also a deterministic way using stable intersections and rational functions as coefficients. Building on techniques similar to [10, Lemma 3.3], one can always find suitable univariate linear polynomials.

We can now state the algorithm to compute $\operatorname{span}(T(I))$.

\section{Algorithm 18}

Input: Generators for an ideal $I \subset \mathbb{K}\left[x_{1}^{ \pm}, \ldots, x_{n}^{ \pm}\right]$.

Output: A vector space basis of $\operatorname{span}(T(I))$.

1. Let $d:=\operatorname{dim}(I)$.

2. If $\operatorname{dim}(I)=0$, then return the basis $\emptyset$ for $\{0\}$.

3. Compute a primitive vector $v \in T(I)$ using Algorithm 16.

4. Compute an invertible matrix $M \in \mathbb{Z}^{n \times n}$ such that $M v=(0, \ldots, 0,1)$.

5. Compute generators of the ideal $I^{\prime}=\phi(I)$ where $\phi: \mathbb{K}\left[x_{1}^{ \pm}, \ldots, x_{n}^{ \pm}\right] \rightarrow \mathbb{K}\left[y_{1}^{ \pm}, \ldots, y_{n}^{ \pm}\right]$ is the multiplicative coordinate change induced by $y_{i}=\prod_{j=1}^{n} x_{j}^{M_{i j}}$.

6. Compute $J^{\prime}=I^{\prime} \cap \mathbb{K}\left[y_{1}^{ \pm}, \ldots, y_{n-1}^{ \pm}\right]$.

7. Recursively compute generators $U$ for $\operatorname{span}\left(T\left(J^{\prime}\right)\right) \subseteq \mathbb{Q}^{n-1}$.

8. Return $\{v\} \cup\left\{M^{-1}(u \oplus(0)): u \in U\right\}$.

\section{The Artinian case}

The proof of Theorem 1 is finished once we describe how to compute ideal generators for the binomial part $\operatorname{Bin}(I)$ of an ideal $I \subset T$ with Artinian quotient $T / I$, where $T=\mathbb{Q}\left[y_{1}^{ \pm}, \ldots, y_{m}^{ \pm}\right]$as above. For $1 \leq i \leq m$, let $M_{i}: T / I \rightarrow T / I$ denote the lin- 
ear endomorphism induced by multiplication with $y_{i}$. With $\ell=\operatorname{dim}_{\mathbb{Q}} T / I$ let $\mathbb{K}$ be the finite extension of $\mathbb{Q}$ which contains the $\ell$ th roots of the determinants of the $M_{i}$. Define $M_{i}^{\prime}=M_{i} / \sqrt[\ell]{\operatorname{det} M_{i}}$. By Remark 8 , it suffices to determine the binomial part of the extension $I \mathbb{K}\left[y_{1}^{ \pm}, \ldots, y_{m}^{ \pm}\right]$. This computation can be translated into a membership problem in the multiplicative group generated by the $M_{i}$.

Proposition 19 Let $e \in \mathbb{Z}^{m}$. There exists a $\lambda \in \mathbb{K}$ such that $\mathbf{y}^{e}-\lambda \in I$ if and only if

$$
\prod_{i=1}^{m}\left(M_{i}^{\prime}\right)^{e_{i}}=\operatorname{Id}_{T / I}
$$

Proof The binomial $\mathbf{y}^{e}-\lambda$ is contained in $I$ if and only if $\prod_{i}\left(M_{i}\right)^{e_{i}}=\lambda \operatorname{Id}_{T / I}$. Taking determinants of both sides yields that in this case $\lambda^{\ell}=\prod_{i}\left(\operatorname{det} M_{i}\right)^{e_{i}}$. So the claim follows from the definition of the $M_{i}^{\prime}$.

The matrices $M_{i}^{\prime}$ commute, are invertible and have entries in a finite extension of $\mathbb{Q}$. In this situation, [1, Theorem 1.2, Section 6.4] gives an algorithm that, for any matrices $M_{1}^{\prime}, \ldots, M_{m}^{\prime}$ with entries in a number field, computes a basis for the lattice of exponents $e \in \mathbb{Z}^{m}$ satisfying (4.1). A more general version is [14, Algorithm 8.3]. Both rely on the LLL lattice basis reduction algorithm.

Remark 20 The commutativity of the matrices is key for algorithmic treatment. For general matrix semigroups, several problems are known to be algorithmically undecidable (see, for example, the table in the end of [9]). In particular, there is no Turing machine program that can decide whether there is a relation among given $(3 \times 3)$ matrices [12]. It is also undecidable if a semigroup generated by eight $(3 \times 3)$ integer matrices contains the zero matrix [18]. This result of Paterson is an important tool to prove other undecidability results. For invertible matrices, group membership is unsolvable for matrices of format $(4 \times 4)$ and larger [16]. Our methods are therefore not directly applicable to polynomials in non-commutative variables.

Finally, the binomial part of the radical of an Artinian ideal $I \subset \mathbb{K}\left[y_{1}^{ \pm}, \ldots, y_{m}^{ \pm}\right]$can be computed without first computing the radical itself.

Proposition 21 For $e \in \mathbb{Z}^{m}$, there exists a $\lambda \in \mathbb{K}$ such that $\mathbf{y}^{e}-\lambda \in \sqrt{I}$ if and only if $\prod_{i=1}^{m}\left(M_{i}\right)^{e_{i}}$ has only one eigenvalue over the algebraic closure $\overline{\mathbb{K}}$. In this case, $\lambda=$ $\prod_{i}\left(\operatorname{det} M_{i}\right)^{e_{i} / \ell}$.

Proof Let $M=\prod_{i=1}^{m}\left(M_{i}\right)^{e_{i}}$. Some power of $\mathbf{y}^{e}-\lambda$ lies in $I$ if and only if $M-\lambda \operatorname{Id}_{T / I}$ is nilpotent. Choose a basis such that $M$ is upper triangular. Then $M-\lambda \operatorname{Id}_{T / I}$ is nilpotent if and only if all entries on the main diagonal of $M$ equal $\lambda$. This equivalent to $M$ having $\lambda$ as its sole eigenvalue. Computing the determinant of $M$ then yields the claimed expression for $\lambda$.

Let $V \subseteq T / I$ be the direct sum of all eigenspaces of $M_{1}$. Then the restriction $\left.M_{1}\right|_{V}$ of $M_{1}$ to $V$ is diagonalizable. Since the $M_{i}$ commute, the same holds for all other $\left.M_{i}\right|_{V}$. Moreover, the set of eigenvalues of $\left.M_{i}\right|_{V}$ equals the set of eigenvalues of $M_{i}$ for each $i$. 
As above, set $M_{i}^{\prime}=M_{i} / \sqrt[\ell]{\operatorname{det} M_{i}}$, where $\ell=\operatorname{dim}_{\mathbb{Q}} T / I$. Let $L \subseteq \mathbb{Z}^{m}$ be the lattice of exponents $e$ that satisfy

$$
\prod_{i=1}^{m}\left(\left.M_{i}^{\prime}\right|_{V}\right)^{e_{i}}=\operatorname{Id}_{V} .
$$

Then $L$ can be computed with the algorithm in [1]. It is clear from the observation above that for each $e \in L$, the matrix $\prod_{i}\left(M_{i}\right)^{e_{i}}$ has only one eigenvalue. So $L$ contains precisely the exponents of the binomials in the radical of $I$.

\section{Algorithm}

To facilitate an implementation of the methods in this paper, we formulate the complete algorithm. In this formulation, the algorithm returns generators of the Laurent extension $\operatorname{Bin}(I) \mathbb{Q}\left[x_{1}^{ \pm}, \ldots, x_{n}^{ \pm}\right]$which, by means of Lemma 5 , yields the desired algorithm for Theorem 1.

\section{Algorithm 22 \\ Input: Generators $f_{1}, \ldots, f_{s}$ for an ideal $I \subset \mathbb{Q}\left[x_{1}, \ldots, x_{n}\right]$ Output: Generators of $\operatorname{Bin}(I) \mathbb{Q}\left[x_{1}^{ \pm}, \ldots, x_{n}^{ \pm}\right]$.}

1. Let $J=\left\langle f_{1}, \ldots, f_{s}\right\rangle \subset \mathbb{Q}\left[x_{1}^{ \pm}, \ldots, x_{n}^{ \pm}\right]$be the Laurent extension of $I$.

2. Compute the orthogonal complement $T(J)^{\perp}$ of the tropical variety ofJ by Algorithm 18.

3. Compute a basis $\left\{b^{(1)}, \ldots, b^{(m)}\right\}$ of the integer lattice $L=T(J)^{\perp} \cap \mathbb{Z}^{n}$. Let $\mathbb{Q}\left[y_{1}^{ \pm}, \ldots, y_{m}^{ \pm}\right]$ with $y_{j}=\mathbf{x}^{b^{(j)}}$ be the resulting Laurent polynomial subring of $\mathbb{Q}\left[x_{1}^{ \pm}, \ldots, x_{n}^{ \pm}\right]$.

4. Compute $K=J \cap \mathbb{Q}[L] \subset \mathbb{Q}\left[y_{1}^{ \pm}, \ldots, y_{m}^{ \pm}\right]$as the preimage of $J$ under the inclusion of $\mathbb{Q}$-algebras $\mathbb{Q}\left[y_{1}^{ \pm}, \ldots, y_{m}^{ \pm}\right] \rightarrow \mathbb{Q}\left[x_{1}^{ \pm}, \ldots, x_{n}^{ \pm}\right]$.

5. Pick a basis of the finite-dimensional $\mathbb{Q}$-algebra $\mathbb{Q}\left[y_{1}^{ \pm}, \ldots, y_{m}^{ \pm}\right] / K$ and compute the matrix representations $M_{i}$ of the linear maps given by multiplication with $y_{i}$.

6. Construct a number field $\mathbb{K}$ that contains all $\ell$ th roots of the determinants of the $M_{i}$, where $\ell:=\operatorname{dim}_{\mathbb{Q}} \mathbb{Q}\left[y_{1}^{ \pm}, \ldots, y_{m}^{ \pm}\right] / K$. Compute $M_{i}^{\prime}=M_{i} / \sqrt[\ell]{\operatorname{det} M_{i}}$

7. Compute a basis $\left\{c^{(1)}, \ldots, c^{(t)}\right\}$ of the lattice $E \subset \mathbb{Z}^{m}$ of exponents satisfying (4.1), for example using the algorithm given in [1].

8. For each $i=1, \ldots, t$, compute $\lambda_{i}$, such that $\mathbf{y}^{c^{(i)}}-\lambda_{i} \in K$, for example, by using $\prod_{j}\left(M_{j}\right)^{c_{j}^{(i)}}=\lambda_{i}$ Id. Then $\operatorname{Bin}(K)=\left\langle\mathbf{y}^{c^{(1)}}-\lambda_{1}, \ldots, \mathbf{y}^{c^{(t)}}-\lambda_{t}\right\rangle \subset \mathbb{Q}\left[y_{1}^{ \pm}, \ldots, y_{m}^{ \pm}\right]$.

9. Return generators of $\operatorname{Bin}(J)$ by substituting $\mathbf{x}^{b^{(j)}}$ for $y_{j}$ in the generators of $\operatorname{Bin}(K)$.

Author details

${ }^{1}$ Aarhus Universitet, Aarhus, Denmark, ${ }^{2}$ Otto-von-Guericke Universität, Magdeburg, Germany, ${ }^{3}$ Goethe-Universität Frankfurt, Frankfurt, Germany.

Acknowledgements

The authors are grateful to Bernd Sturmfels for suggesting to approach the problem through tropical geometry and for constant encouragement. The application of tropical geometry to binomial containment was pointed out to the first author by Douglas Lind. Alice Silverberg and Hendrik W. Lenstra provided valuable help in navigating the computational number theory literature, in particular, locating a copy of [7].

\section{Publisher's Note}

Springer Nature remains neutral with regard to jurisdictional claims in published maps and institutional affiliations. 
References

1. Babai, L., Beals, R., Cai, J-y., Ivanyos, G., Luks, EM.: Multiplicative equations over commuting matrices. In: Proceedings of the 7th Annual ACM-SIAM Symposium on Discrete Algorithms (Atlanta, GA, 1996), pp. 498-507. ACM, New York (1996)

2. Bogart, T., Jensen, A.N., Speyer, D., Sturmfels, B., Thomas, R.R.: Computing tropical varieties. J. Symb. Comput. 42(1-2), 54-73 (2007)

3. Bruns, W., Gubeladze, J.: Polytopal linear retractions. Trans. Am. Math. Soc. 354(1), 179-203 (2002)

4. Chan, A.: Gröbner Bases Over Fields with Valuations and Tropical Curves by Coordinate Projections, Ph.D. thesis, University of Warwick (2013)

5. Conradi, C., Kahle, T.: Detecting binomiality. Adv. Appl. Math. 71, 52-67 (2015)

6. Eisenbud, D., Sturmfels, B.: Binomial ideals. Duke Math. J. 84(1), 1-45 (1996)

7. Ge, G.: Algorithms Related to Multiplicative Representations, Ph.D. thesis, University of California, Berkeley (1993)

8. Greuel, G.-M.: A Singular Introduction to Commutative Algebra, 2nd edn. Springer, New York (2007)

9. Halava, V.: Decidable and Undecidable Problems in Matrix Theory, Turku Centre for Computer Science (1997)

10. Jensen, A.N., Yu, J.: Stable intersection of tropical varieties. J. Algebr. Comb. 43(1), 101-127 (2016)

11. Kahle, T., Miller, E.: Decompositions of commutative monoid congruences and binomial ideals. Algebra Number Theory 8(6), 1297-1364 (2014)

12. Klarner, D.A., Birget, J.-C., Satterfield, W.: On the undecidability of the freeness of integer matrix semigroups. Int. J. Algebra Comput. 1(2), 223-226 (1991)

13. Kreuzer, M., Robbiano, L.: Computational Commutative Algebra 2. Springer, New York (2005)

14. Lenstra, H., Silverberg, A.: Algorithms for Commutative Algebras Over the Rational Numbers, Foundations of Computational Mathematics (2016), To appear

15. Maclagan, D., Sturmfels, B.: Introduction to Tropical Geometry, Graduate Studies in Mathematics, vol. 161. AMS, Providence, RI (2015)

16. Mihălova, K.A.: The occurrence problem for direct products of groups. Dokl. Akad. Nauk SSSR 119(6), 1103-1105 (1958)

17. Miller, E.: Finding All Monomials in a Polynomial Ideal, Preprint (2016)

18. Paterson, M.S.: Unsolvability in $3 \times 3$ matrices. Stud. Appl. Math. 49(1), 105-107 (1970)

19. Saito, M., Sturmfels, B., Takayama, N.: Gröbner Deformations of Hypergeometric Differential Equations, Algorithms and Computation in Mathematics, vol. 6. Springer, Berlin (2000)

20. Sontag, E.D.: A technique for determining the signs of sensitivities of steady states in chemical reaction networks. IET Syst. Biol. 8(6), 251-267 (2014)

\section{Submit your manuscript to a SpringerOpen ${ }^{\circ}$} journal and benefit from:

$\rightarrow$ Convenient online submission

- Rigorous peer review

- Immediate publication on acceptance

- Open access: articles freely available online

- High visibility within the field

- Retaining the copyright to your article

Submit your next manuscript at springeropen.com 